\title{
Numerical Simulation and Experimental Study on Parameter Optimization of Cold Flanging Process of Pipe Tee
}

\author{
Gang Cheng ${ }^{1, a}$, Shiao Zhang ${ }^{1, b}$, Xiuqing $\mathrm{Ma}^{2}$, Yunjiang $\mathrm{Li}^{1}$, Guoqun Zhao ${ }^{3}$, \\ Qiang Cheng ${ }^{4}$, Zhonglei Wang ${ }^{1}$ and Teng $\mathrm{He}^{1}$ \\ ${ }^{1}$ School of mechanical and electronic engineering, Key Laboratory of Mechanical Engineering \& \\ Innovation Technology, Shandong Jianzhu University, Jinan250101, China \\ ${ }^{2}$ School of Civil Engineering, Shandong University, Jinan 250061, China \\ ${ }^{3}$ Engineering Research Center of Mould \& Die, Shandong University , Jinan 250061, China \\ ${ }^{4}$ School of Mechanical and Automotive engineering, Qilu University of Technology, Jinan 250353, \\ China

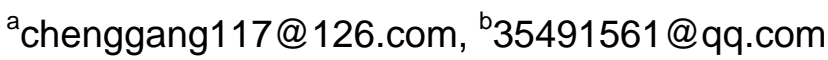

Keywords: Pipe tee, Prefabricated holes, Cold flanging, Parameter optimization.

Abstract. Tee flange molding is a very complex process of change. The affect of the preformed holes is very large in the molding process, so we should calculate according to the parameters and Simulation by Dynaform in order to find the place of torn or wrinkled during the molding process . Then we can not only guarantee that the branch pipes is not wrinkled and torn but also can improve the smoothness and height of the branch pipes by optimizing of the shape of the prefabricated holes.

\section{Introduction}

In this era where industry is developing fast, the application of multi-branch tubes is very broad; especially the three-limb tubes, whose application in each field is increased constantly, and this can be reflected in the transportation of petroleum and natural gas in particular.

All manuscripts must be in English, also the table and figure texts, otherwise we cannot publish your paper.

There are many traditional processing methods for the three-limb tubes, such as forging and direct welding. However, along with the performance requirements of three-limb tubes in the application of each field, these methods can no longer meet the demand. Size of the forged three-limb tubes is relatively large, and lots of materials will be cut off during the processing, therefore, it is of more steel consumption amount and more complicated technology; the traditional three-limb branched pipe welding is to cut the corresponding intersection-line hole and end face at the joint of main pipe and branched pipe, and then solder them together. This production method is relatively difficult for processing the intersection-line hole and end face, and besides that, its demand of space welding technology is quite high, and therefore, automatic production and the processing accuracy are hard to achieve and ensured [1]. The processing technology we studied is to firstly process a prefabricated hole on the main pipe, and then process an outlet flange through flanging technology, and directly solder the branched pipe to the flange. This method is not only of simple welding technology, but also of high accuracy, realizing automatic production and improving mechanical property of the three-limb tubes [2]. During the cold flanging forming process of the three-limb tubes, there are many factors that might influence the effect [3], including the calculation and optimization of the prefabricated hole, the design of testing mould, the selection of terrace die and the drawing speed during the test. This paper is mainly focused on the introduction of the prefabricated hole calculation during the cold flanging forming process of the three-limb tubes [4-5], its optimization and the corresponding finite element simulation [6], and then further optimizing the prefabricated hole by analog simulation, and obtaining more perfect experimental results. 


\section{Calculation of the prefabricated hole of equal-diameter three-limb tubes}

Generally, there are two kinds of computing methods for calculating the prefabricated hole of plane round-hole flanging technology, namely, the equal conductor length method and the equal area method, of which, the equal conductor length method holds that flanging technology is similar to the bending technology. Assuming that length of the tessellation lines in the radial direction of ring-shaped deformation area around the round hole remains unchanged, it is believed that the deformation during the flanging forming process mainly relies on the thinning of material; and in terns of the equal area method, it holds that thickness remains unchanged during the flanging process. Assuming that areas of the material before and after the flanging are equal, it is believed that the deformation during the flanging forming process mainly relies on changes of the length of tessellation lines in the radial direction of ring-shaped deformation area.

In terms of the plane round-hole flanging as shown in Fig. 1, the equal conductor length theory shall be adopted, namely, radius of the circular ring before flanging forming is equal to the sum of lengths of circular bead and vertical wall, and thus solution formula of the prefabricated hole can be obtained

$$
d_{0}=D_{m}+0.86 r+1.43 t-2 H
$$

Wherein, $\mathrm{d}_{0}$ is the diameter of prefabricated hole; $\mathrm{D}_{\mathrm{m}}$ is the diameter of neutral layer of the flanged hole; $r$ is the fillet radius formed by flanging; $\mathrm{H}$ is the flanging height; $\mathrm{t}$ is the thickness of the flanging material.

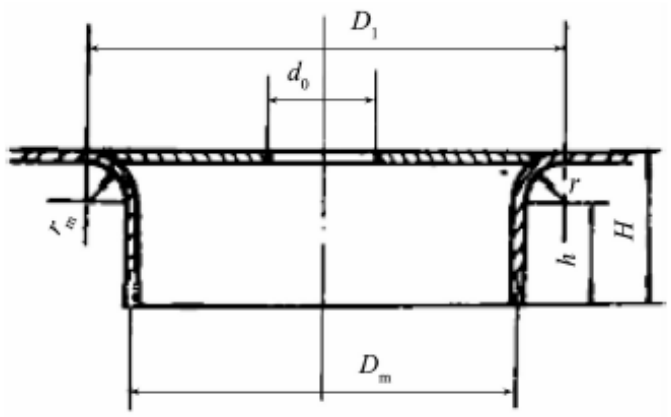

Fig. 1 Plane round-hole flanging

\section{Formula derivation of the sizes of prefabricated hole}

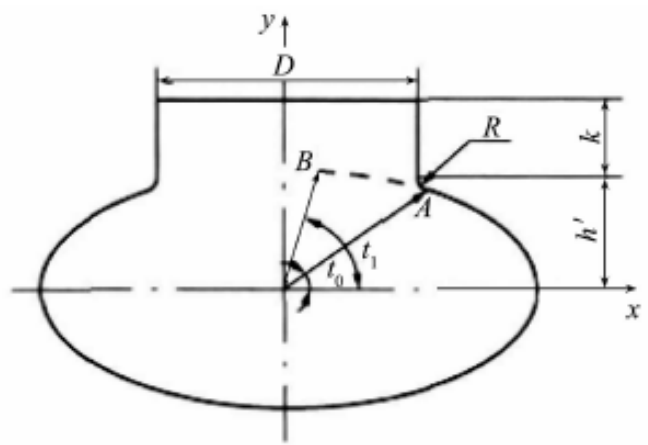

Fig. 2 Cross-section through the branched pipe axis along an arbitrary plane

Calculation of the form of prefabricated hole of equal-diameter three-limb tubes is very complicated, and the branched pipe and main pipe can be cut out along the plane of branched pipe axis that forms an arbitrary inclined angle of $\theta$ with the $\mathrm{X}$ axis, and the results are as shown in Fig. 2. Cross-section of main pipe is a standard ellipse, of which, the semi-major axis is $D / 2 \sin \theta$, and minor semi-axis is $D / 2$, of which, $0 \leq \theta \leq \pi / 2$; in case that $\theta=0$, length of the joint arc is $\pi R / 2$; in case that $\theta=\pi / 2$, the arc length is 0 . It is required that the branched pipe port shall be smooth during the flanging; assuming that the flanging height is $h$, and then height of the vertical wall of branched pipe is $h+D / 2$.

In the coordinate system of cross-section, parameter equation of the ellipse is:

$$
x=\frac{D \cos t}{2 \sin \theta}, y=\frac{D \sin t}{2}
$$

It can be seen from Fig. 2 that

$$
h+D / 2=b^{\prime}+k
$$

Wherein, $b^{\prime}=y+\sqrt{R^{2}-(x-D / 2-R)^{2}},(x, y)$ refers to any point in the ellipse, and $t$ refers to the angle corresponding to this point.

Therefore, we can get,

$$
k=D / 2+h-\left(y+\sqrt{R^{2}-(x-D / 2-R)^{2}}\right)
$$


Thus, the deformed length of the branched pipe after flanging equals to $k$ plus the arc length of circular bead, i.e.,

$$
L=k+R \cdot \frac{\pi}{180} \arcsin \frac{\sqrt{R^{2}-(x-D / 2-R)^{2}}}{R}
$$

Based on the principle of unchanged length of tessellation lines in the radial direction of ring-shaped deformation area before and after the deformation, it can be believed that length of the deformation area (L) is obtained by the bending of $\mathrm{AB}$ on the ellipse; assuming the length of $\mathrm{AB}$ is $\widehat{S}$, and thus, the arc $\widehat{S}=L$, i.e.,

$$
\widehat{S}=\int_{t_{0}}^{t_{1}} \sqrt{\left(\frac{D \sin t}{2 \sin \theta}\right)^{2}+\left(\frac{D \cos t}{2}\right)^{2}} d t=L
$$

$t_{0}$ and $t_{1}$ can be obtained by adopting the numerical integration method, and thus $x_{\theta}$ can be obtained. Therefore, when angle of the cross-section is $\theta$, coordinate of Point $\mathrm{B}$ is $\left(x_{\theta}, y_{\theta}\right)$. After $\left(x_{\theta}, y_{\theta}\right)$ of each cross-section has been defined, coordinate of the prefabricated hole can be defined.

\section{Numerical simulation of three-dimensional finite element}

\section{Research object and methods of the numerical simulation}

Research object of this paper is the equal-diameter three-limb tube with external diameter of $112 \mathrm{~mm}$, wall thickness of $2 \mathrm{~mm}$, pipe fitting length of $200 \mathrm{~mm}$, drawing height (h) of $8 \mathrm{~mm}$ and circular bead (R) of $4 \mathrm{~mm}$; the drawing temperature is ambient temperature and the working speed of the tensile machine is $15 \mathrm{~mm} / \mathrm{s}$. In this simulation, analog simulation of the drawing process is performed by adopting Dynaform software, to test whether the flatness and drawing height of the branched pipe port are qualified after the draw forming. Since the mathematical model established in this paper is quite complicated, with integral equation being included. It is very hard to calculate. Therefore, Delphi programming system is adopted to solve the question correctly and conveniently, and meanwhile, solving program of mathematical model is developed, and then by combining the calculation formula of prefabricated hole, one group of size date thereof can be obtained, which is as shown in Table 1.

As per the data of prefabricated hole, the opening on the main pipe is as shown in Fig. 3, and as per the experimental requirements, Dynaform software is adopted for the analog simulation, and the results are as shown in Fig. 4.

Table. 1 Primary computing data of the prefabricated hole

\begin{tabular}{ccccccccccc}
\hline Angle $/\left(^{\circ}\right)$ & 0 & 10 & 20 & 30 & 40 & 50 & 60 & 70 & 80 & 90 \\
\hline$X / \mathrm{mm}$ & 51.1 & 49.4 & 44.6 & 37.5 & 29.5 & 21.9 & 15.8 & 10.0 & 5.14 & 0 \\
$y / \mathrm{mm}$ & 0 & 8.6 & 16.0 & 21.4 & 24.4 & 25.6 & 25.3 & 25.3 & 24.5 & 24.5 \\
\hline
\end{tabular}

It can be seen from Fig. 4 that there is no crack on the branched pipe port, and no puckering phenomenon at joints of the branched pipe and the main pipe, indicating that size of the prefabricated hole is reasonably good, however, it is easy to see that the flanging height and flatness of the flange port that is drew out are not so good, which will bring unnecessary troubles during the welding of branched pipe, and meanwhile, influence the accuracy of welding as well as the overall quality of the three-limbs tube. To improve the flanging height and the flatness of the flange port, the most important thing is to optimize the geometrical size of the prefabricated hole.

\section{Optimization of the prefabricated hole}

Since the equal conductor length theory is more consistent with the actual conditions, the equal conductor length theory is taken as the basis for the optimized design of this paper, to perform the 
optimized design of the prefabricated hole. As per the equal conductor theory, linear size of the deformation material before and after the flanging forming remains unchanged, and therefore, in the same direction, the changes of flanging height equal to the changes of radial length. Through calculating the difference value of the actual flanging height of optional position and the ideal height thereof, the difference value of the actual size of prefabricated hole and the ideal size thereof can be obtained, and thus more ideal prefabricated hole can be calculated. To obtain more accurate size of the prefabricated hole, multi-iterations can be performed, to calculate more ideal size of prefabricated hole. The top view thereof is as shown in Fig. 5, and now, derivation of formula can be obtained: firstly, define $\Delta H$ as the difference value of the flanging height after simulation (H1) and the ideal height (h), i.e., $\Delta H=H_{1}-h$.

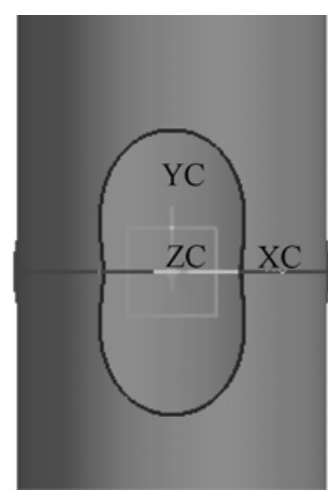

Fig. 3 Prefabricated hole

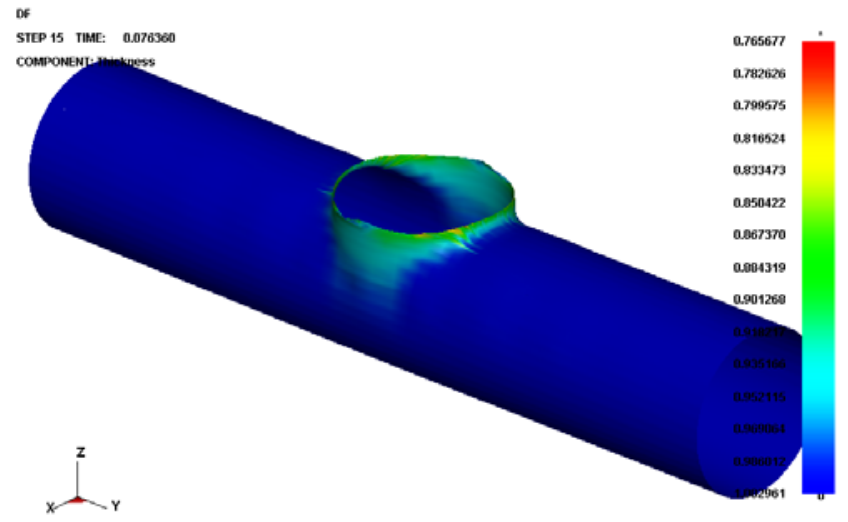

Fig. 4 Simulation of pipe fitting forming

Assuming that $\Delta L=0.8 \times \Delta H$, and then it can be seen from the figure that

$$
X_{2}=X_{1}+\Delta L \times \cos \theta, Y_{2}=Y_{1}+\Delta L \times \sin \theta
$$

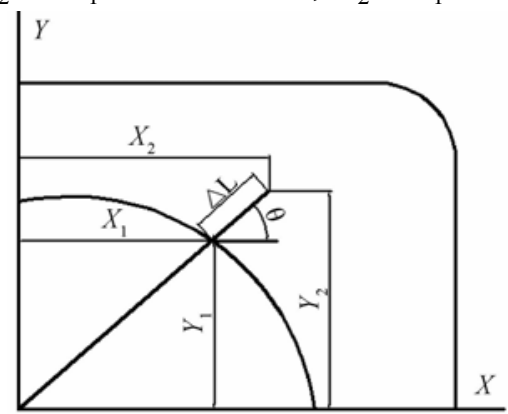

Fig. 5 Top view of the optimized prefabricated hole

As per a series of iterative calculation, optimized sizes of the prefabricated hole can finally be obtained as shown in Table 2.

Tab. 2 Data of the prefabricated holes after the optimization

\begin{tabular}{rcccccccccc}
\hline Angle $/\left(^{\circ}\right)$ & 0 & 10 & 20 & 30 & 40 & 50 & 60 & 70 & 80 & 90 \\
\hline $\mathrm{x} / \mathrm{mm}$ & 48.7 & 46.0 & 40.5 & 33.3 & 25.8 & 18.4 & 12.7 & 8.1 & 3.9 & 0 \\
$\mathrm{y} / \mathrm{mm}$ & 0 & 8.0 & 14.6 & 19.0 & 21.4 & 22.3 & 22.3 & 22.6 & 22.6 & 22.7 \\
\hline
\end{tabular}

As per the data of prefabricated hole, the opening on the main pipe is as shown in Fig. 6, and as per the experimental requirements, Dynaform software is adopted for the analog simulation, and the results are as shown in Fig. 7.

It can be seen from Fig. 7 that, in terms of the forming quality, simulated results of this time are much better than that before the optimization of prefabricated hole, and there is no crack or puckering phenomenon appears; meanwhile, the flanging height is increased by $2 \mathrm{~mm}$, and the flange port is relatively smooth. 


\section{Experimental results}

Perform flanging experiment towards the above two kinds of pipe fittings with different sizes by adopting the pressure testing machine, and the results are as shown in Fig.8, and the experimental results are rather obvious; the experimental result of the optimized prefabricated hole is the same with the simulated result, and no matter it is the flanging height or the flatness of the pipe port, large improvements can be seen when being compared with experimental results of the original prefabricated hole.

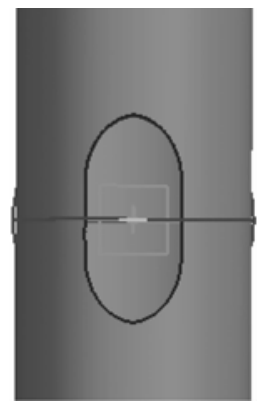

Fig. 6 Prefabricated hole

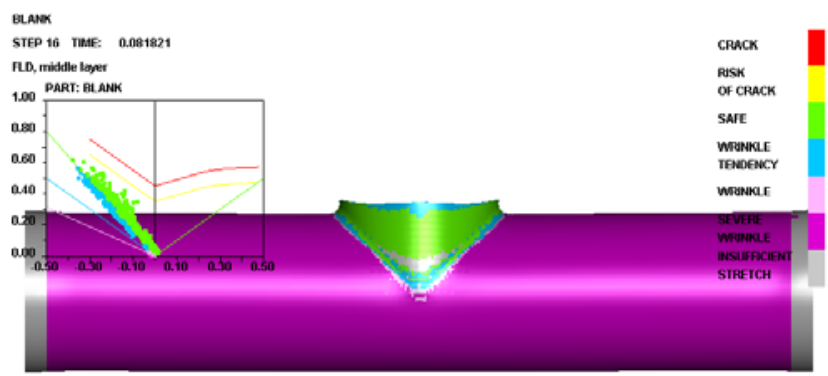

Fig. 7 Simulation of pipe fitting forming

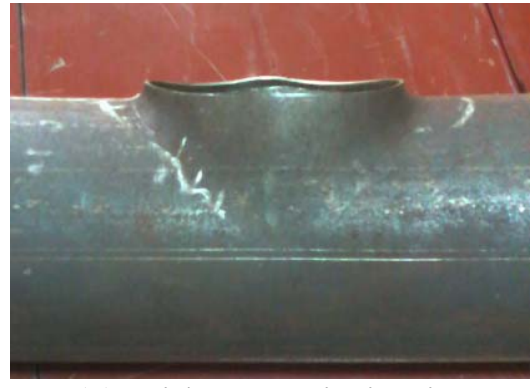

(a) without optimization

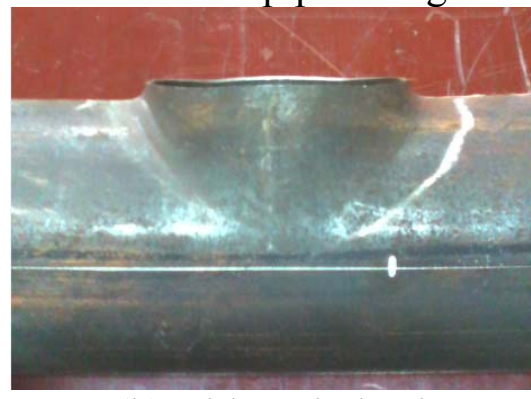

(b) with optimization

Fig. 8 Experimental results of prefabricated holes

\section{Conclusions}

Through the calculation, optimized analysis and finite element simulation of the equal-diameter three-limb tubes with inner diameter of $112 \mathrm{~mm}$ as well as lots of experiment, this paper has obtained three-limb tubes with different forming qualities, and then by comparing the forming quantity of the branched pipe, conclusions are drawn as follows:

1) Computing method of the cold flanging fabricated hole is performed as per the pressing and deforming rules, and it has been verified through mathematical analytical reasoning that, adoption of this method is not only of theoretical basis, but also enables numerical calculation to be performed by using the computer.

2) By adopting iterative method, optimize the prefabricated hole calculated by equal conductor length theory, and the optimized results will not only improve the height of the branched pipe, but also enable the branched pipe port to be smoother, and the non-uniformity in thickness can be largely reduced.

3) Through the experimental study of the flanging technology of three-limb tubes, we can find that, for three-limb tubes with specific sizes, the one-off forming of prefabricated hole that is designed by the pressing and deforming rules is feasible.

\section{References}

[1] M. J. Ni: Electric Welding Machine Vol. 31 (2001), p.37 ( In Chinese) .

[2] S. Y. Luo: Petro-Chemical Equipment Vol. 22 (1993), p.43 (In Chinese) .

[3] X. P. Wang: Stamping Manual(China Machine Press, Beijing 2004) (In Chinese) . 
[4] Z. Qin, X. Li, X. L. Zhao and Z.L.Wang: Science \& Technology Information Vol. 22 (2013), p. 456 ( In Chinese) .

[5] H. S. Wang: Hot Working Technology, Vol.38 (2009), p.86 ( In Chinese) .

[6] L.M. Liu, Z.P. Zhong and T. Xie: Journal of Plasticity Engineering Vol. 16 (2009), p.15 ( In Chinese) . 